\title{
A Method of Analyzing Density-Dependent Vital Rates With an Application to the Gainj of Papua New Guinea
}

\author{
JAMES W. WOOD AND PETER E. SMOUSE \\ Department of Human Genetics, University of Michigan Medical School, Ann \\ Arbor, Michigan 48109
}

\author{
KEY WORDS Demography, Density-dependence, Population \\ regulation, New Guinea
}

\begin{abstract}
A method of estimating age-specific coefficients of density-dependent variation in fertility and mortality is developed; the method is applicable to longitudinal data on population size and the number of births and deaths classified by age. Given a sufficiently large data set, it is possible to estimate both the sensitivity of each age class to density-dependent damping and the density effect of each age class on every age class in the population. Application of the method to government census data on the Gainj, a small tribal population from highland Papua New Guinea, shows that fertility is density-independent, but that mortality is at least partially density-dependent. This finding suggests that the size of the population is regulated by mortality rather than fertility. Individuals aged less than five years and greater than 50 years are particularly sensitive to density-dependent survival damping; individuals of adolescent and early reproductive age are not themselves damped, but appear to be responsible for the observed damping.
\end{abstract}

It is a common assertion that small human populations at the band or tribal level maintain their numbers in stable equilibrium through some form of population regulation. Although there is a good deal of anecdotal evidence in favor of this view, we know of no rigorous quantitative demonstration that it is true of any human population. For a direct demonstration of population regulation, it would be necessary to establish two separate facts: First, an equilibrium actually exists, so that the size of the population in question is effectively constant over some protracted period of time. Second, the equilibrium is stable, so that the population returns to the equilibrium after being displaced from it. Unfortunately, it would take many years of observation to develop this sort of direct demonstration, and the special circumstances under which both convergence to equilibrium and the equilibrium itself can be observed are probably rare. It can be shown under realistic assumptions, however, that a necessary and sufficient condition for the existence of a stable demographic equilibrium is that the growth rate of the population is a decreasing function of population size (Leslie, 1959; Pielou, 1977; Charlesworth, 1980). This condition is known as negative density-dependence, since it implies that population growth is negatively correlated with population size or density. In general, it doubtless takes far fewer years of observation to detect a relationship of negative density-dependence than to construct a convincing direct demonstration of stable demographic equilibrium.

If it can be established that population growth is negatively density-dependent, at least two additional questions must be answered before anything can be inferred about the mechanisms of population regulation. First, how do the various age groups that make up the population contribute to density-dependent damping? That is, are the vital rates of any given age group sensitive to damping, and do changes in the size of the age group provoke changes in vital rates either for that same age group

Received August 17, 1981; accepted February 11, 1982. 
or for any other age group? Second, what are the relative roles played by fertility and mortality in damping population growth? This question arises because the condition of negative density-dependence can be met either by the birth rate declining or the death rate increasing (or both) as the population grows in size. Most anthropologists appear to believe that population regulation, if it occurs in humans at all, is mediated through some form of fertility control. (For recent examples, see Benedict, 1972; Hayden, 1972; Dumond, 1975; Halberstein, 1980). This view is based on evidence that various mechanisms, either cultural or physiological, act to lower fertility below some theoretical maximum. To regulate the population, however, fertility must be adjustable in a negative density-dependent fashion, at least in the neighborhood of demographic equilibrium. If fertility is low but constant, it cannot regulate the population. The same argument applies to mortality, even though mortality is rarely invoked by anthropologists as a mechanism of population regulation.

In this paper, we develop new methods of fitting and testing models of age-specific, density-dependent fertility and mortality, and we apply these methods to census data from a small tribal population, the Gainj of highland Papua New Guinea. Here we are particularly concerned with developing the necessary statistical tools for this analysis, with showing that density-dependent damping of population growth actually occurs among the Gainj, and with investigating the relative sensitivities of the fertility and mortality of various age groups to this damping. In a sequel to this paper we shall deal with the implications of such damping for the population's age structure and the form of the demographic equilibrium itself.

\section{MODELS OF DENSITY.DEPENDENT DAMPING}

We begin with a description of the basic models to be tested. All these models can be derived from a simple modification of the density-dependent model of Smouse and Weiss (1975). The essence of that earlier treatment is that both the probability of surviving and the probability of reproducing during a short interval of time are log-linear functions of the age distribution and size of the population at the beginning of the interval. For convenience, we shall describe only models of mortality in this section. Structurally equivalent models of fertility can also be constructed, and we shall test such models later in the paper. In conformity with standard demographic practice, we adopt a female-dominant, single-sex treatment here. This greatly simplifies the mathematics and, as we shall discuss below, is particularly appropriate for the application to follow.

Let the population be divided into $I$ nonoverlapping age classes. For a female in the $i$-th age class at time $t$, let the probability of surviving one time unit (say, one year) be denoted by $p_{i t}(i=1,2, \ldots, I ; t=1,2, \ldots, T)$. (Similarly, in our models of fertility we work in terms of $f_{i t}$, the probability that a female in the $i$-th age class produces a single daughter in the same interval.) The number of females present in the age class at the beginning of the interval is denoted by $n_{i t}$. Following the Smouse-Weiss model, we define

$$
\ln p_{i t}=\alpha_{i}-\sum_{j=1}^{I} \beta_{i j} n_{j t}=\alpha_{i}-\boldsymbol{\beta}_{i} \mathbf{n}_{t}
$$

to be the density-dependent survival probability for the $i$-th age-class during the $t$-th census period. The term $\alpha_{i}$ is the density-independent component of survival in the $i$-th age class. (The traditional density-independent demographic treatment describes $p_{i t}$ by the simpler model $\ln p_{i t}=\alpha_{i}$, so that $p_{i t}$ is a constant that does not depend on population composition.) The $\beta_{i j}$ are positive "damping coefficients" that determine the influence of the $j$-th age class on the survival of the $i$-th age class. Given these negatively density-dependent survival rates or a parallel model for fertility, it is easily shown that the population converges to both a stable age distribution and an equilibrium population size under all but the most extreme demographic circumstances (cf. Smouse and Weiss, 1975).

Because exponents are additive, the exponential form of eq. (1) is mathematically convenient for use with Leslie projection matrices; however, it involves two serious problems with respect to estimation. First, nothing in it constrains $p_{i t}$ to lie between zero and one. Second, it does not produce a smooth sigmoid curve of the sort usually assumed to characterize density-dependent processes. A very similar model that avoids both of these difficulties is provided by the familiar logit transformation:

$$
\begin{gathered}
\Omega: \quad \ln \left(\frac{p_{i t}}{1-p_{i t}}\right) \\
=\alpha_{i}-\sum_{j=1}^{I} \beta_{i j} n_{j t}=\alpha_{i}-\boldsymbol{\beta}_{i} \mathbf{n}_{t} .
\end{gathered}
$$


The parameters here have exactly the same meanings as before, but the model is more useful for estimation. For either large $\left(0.80<p_{i t}\right.$ $<1.00)$ or small $\left(0.00<p_{i t}<0.20\right)$ survival probabilities, eqs. (1) and (2) yield similar $\beta$ coefficients. Only in the intermediate range $\left(0.20<p_{i t}<0.80\right)$ do the $\beta$-coefficients differ appreciably between these models, and we are not operating in this range.

The full model $(\Omega)$ requires $I(I+1)$ parameters for survival and a similar number for fertility; it is thus both completely general and theoretically rich. Unfortunately, the quantity of data required to estimate so many parameters is prohibitive for most anthropologically interesting situations; the full model is a bit too rich to be of much utility. Therefore we present a series of simplified models that retain various important features of the most general model (cf. Weiss and Smouse, 1976).

The first such simplification, Model I, is the density-independent model, which will serve as our null hypothesis:

$$
\text { I: } \begin{aligned}
\ln \left(\frac{p_{i t}}{1-p_{i t}}\right) & =\alpha_{i} \\
\quad \text { or } p_{i t} & =\frac{\mathrm{e}^{\alpha_{i}}}{1+\mathrm{e}^{\alpha_{i}}}=p_{i .}
\end{aligned}
$$

The second simplification, Model II, allows for generalized damping by total population size $(n \cdot t)$ :

$$
\text { II: } \quad \ln \left(\frac{p_{i t}}{1-p_{i t}}\right)=\alpha_{i}-\gamma_{i} n \cdot t .
$$

Finally, the third simplification, Model III, is identical to the most general model $(\Omega)$, except that it assumes that some of the $\beta$-coefficients are zero. Given a subset $(H \subset I)$ of the $n_{h t}$ assumed to damp, Model III takes the form

$$
\text { III: } \begin{gathered}
\ln \left(\frac{p_{i t}}{1-p_{i t}}\right) \\
=\alpha_{i}-\sum_{h \in I}^{H \subset I} \beta_{i h} n_{h t}=\alpha_{i}-\boldsymbol{\beta}_{i}^{* \prime} \mathbf{n}_{t}^{*}
\end{gathered}
$$

where the summation is over that same subset $(H)$ of the $I$ age classes. Models I, II, III and $\Omega$ require $I, 2 I, I(H+1)$ and $I(I+1)$ parameters, respectively.

We should note at this point that all these models have constant parameters and thus are not applicable to cases undergoing demographic transition due to social or environmental change. In any particular application, it will be necessary to find some sort of transient perturbation that changes the size of the population enough to reveal density effects without modifying its underlying demographic patterns.

\section{ESTIMATION AND TESTING}

Our estimation strategy, though superficially complicated, is basically a weighted leastsquares procedure. As before, we find it convenient to treat only mortality here, but the procedures for fertility are precisely analogous.

Although we do not actually use the full model (eq. 2) here, we begin with the estimation of its parameters since estimates for all the other models can then be obtained as a series of special cases. Observed survival fractions of 1 and 0 create statistical problems, because one cannot take the logarithm of 0 . Gart and Zweifel (1967) have shown that this problem can be circumvented and the chi-square approximations improved if one defines (in our terminology)

$$
y_{i t}=\ln \left[\frac{p_{i t}+1 /\left(2 n_{i t}\right)}{1-p_{i t}+1 /\left(2 n_{i t}\right)}\right] .
$$

We then have the linear model

$$
y_{i t}=\alpha_{i}-\boldsymbol{\beta}_{i}^{\prime} \mathbf{n}_{t}+\boldsymbol{\epsilon}_{i t}
$$

Gart and Zweifel (1967) have also shown that the variance of the error term $\epsilon_{i t}$ is approximately

$$
\operatorname{Var}\left(\epsilon_{i t}\right)=\frac{\left(n_{i t}+1\right) / n_{i t}^{2}}{\left[p_{i t}+1 /\left(2 n_{i t}\right)\right]\left[1-p_{i t}+1 /\left(2 n_{i t}\right)\right]}
$$

Now define the vectors $\boldsymbol{\theta}_{i}^{\prime}=\left(\alpha_{i}, \beta_{i 1}, \beta_{i 2}, \ldots\right.$, $\left.\beta_{i l}\right)$ and $\mathbf{x}_{t}=\left(1,-n_{1 t},-n_{2 t}, \ldots,-n_{I t}\right)$. The general model can be rewritten

$$
y_{i t}=\alpha_{i}-\boldsymbol{\beta}_{i}^{\prime} \mathbf{n}_{t}+\epsilon_{i t}=\boldsymbol{\theta}_{i}^{\prime} \mathbf{x}_{t}+\boldsymbol{\epsilon}_{i t} .
$$

Place the observed $y_{i t}$ values into a vector $\mathbf{y}_{i}^{\prime}$ $=\left(y_{i 1}, y_{i 2}, \ldots, y_{i T}\right)$ and construct a matrix $\mathbf{X}$ of dimension $T \times(I+1)$ whose $t$-th row is the observed vector $\mathbf{x}_{t}$. Define the inverse-variance weights $w_{i t}=1 / \operatorname{var}\left(\epsilon_{i t}\right)$ using eq. (7) and place them in a diagonal matrix $\mathbf{W}_{i}$ of dimension $T$ 
$\times T$. The coefficient vector, $\boldsymbol{\theta}_{i}$, can now be estimated according to a standard weighted leastsquares normal equation (Neter and Wasserman, 1974: 327),

$$
\boldsymbol{\theta}_{i}=\left(\mathbf{X}^{\prime} \mathbf{W}_{i} \mathbf{X}\right)^{-1}\left(\mathbf{X}^{\prime} \mathbf{W}_{i} \mathbf{y}_{i}\right) .
$$

According to the Gauss-Markov theorem, this is the best estimator of $\theta_{i}$ in that it is unbiased and of minimum variance among all weighted estimates (Rao, 1973). A separate vector $\theta_{i}$ is estimated for each age class, and the $y_{i t}$ predicted by the model are computed as

$$
\begin{aligned}
\hat{y}_{i t}=\hat{\boldsymbol{\theta}}_{i} \mathbf{x}_{t}=\hat{\alpha}_{i} & -\hat{\beta}_{i 1} n_{1 t} \\
& -\hat{\beta}_{i 2} n_{2 t}-\ldots-\hat{\boldsymbol{\beta}}_{i I} n_{I t} .
\end{aligned}
$$

Define a vector of these $\hat{y}$ values for the $i$-th age group, viz., $\hat{\mathbf{y}}_{i}^{\prime}=\left(\hat{y}_{i 1}, \hat{y}_{i 2}, \ldots, \hat{y}_{i T}\right)$.

The goodness of fit of the general model is assessed by partitioning the total variation in the observed $y_{i t}$ values into a component attributable to the model and a residual component. For the $i$-th age class, we compute the following weighted sums of squares:

$$
\begin{aligned}
S S(\text { Total }) & =\mathbf{y}_{i}^{\prime} \mathbf{W}_{i} \mathbf{y}_{i}=\sum_{t} w_{i t} y_{i t}^{2} \\
S S(\text { Model } \Omega) & =\mathbf{y}_{i} \mathbf{W}_{i} \mathbf{X} \hat{\mathbf{\theta}}_{i} \\
S S(\text { Residual } \Omega) & =\left(\mathbf{y}_{i}-\hat{\mathbf{y}}_{i}\right)^{\prime} \mathbf{W}_{i}\left(\mathbf{y}_{i}-\hat{\mathbf{y}}_{i}\right) \\
& =\sum_{t} w_{i t}\left(y_{i t}-\hat{y}_{i t}\right)^{2}
\end{aligned}
$$

The $y_{i t}$ values are defined so that $S S($ Model $\Omega$ ) and $S S($ Residual $\Omega$ ) are distributed as chi-square variables with $(I+1)$ and $(T-I-1)$ degrees of freedom, respectively. Obviously, to estimate all the parameters we require $T>I+$ 1 , and in general we need $T>>I$ to achieve satisfactory estimates. Exactly analogous treatment is possible for fertility, but since only, say, $K<I$ age classes will usually be fertile, we need only $T>K+1$ to estimate all the non-zero fertility parameters. If $T$ (the number of separate censuses) is too small for the full estimation task-and in the following application it will be too small - one must be content with one of the simpler models, I, II, or III.

One usually prefers to reduce the number of parameters to a parsimonious minimum that still allows an adequate fit of the model to the data. One begins with the simplest version, Model I, and adds only those terms required to improve the degree of fit. For Model I, the es- timators and sums of squares can be written down immediately:

$$
\begin{aligned}
\hat{y}_{i t} & =\frac{\sum_{t} w_{i t} y_{i t}}{\sum_{t} w_{i t}}=\bar{y}_{i .} \\
\text { SS(Model I) } & =\sum_{t} w_{i t} \bar{y}_{i}^{2} . \\
S S(\text { Residual I }) & =\sum_{t} w_{i t}\left(y_{i t}-\bar{y}_{i}\right)^{2} .
\end{aligned}
$$

Since Model I is the null hypothesis of densityindependence, eq. (13b) is the total variation available for modeling density-dependent deviations. If eq. (13b) is not significant when compared to a chi-square distribution with ( $T$ - 1) degrees of freedom, then there is no evidence for density-dependence and the analysis should terminate.

If Model I does not provide an adequate fit to the data, we then assess Model II. The estimation and testing strategy is exactly the same as in eqs. (9-11), except that the $\theta_{i}$ and $\mathbf{x}_{t}$ vectors now take the form $\boldsymbol{\theta}_{i}^{\prime}=\left(\alpha_{i}, \gamma_{i}\right)$ and $\mathbf{x}_{t}^{\prime}=\left(1,-n_{t}\right)$, where $n_{t}$ is the total population size at the beginning of the $t$-th census period. The fit of Model II is assessed by computing

$$
\begin{aligned}
S S(\text { Model II })= & S S(\text { Residual I }) \\
& -S S(\text { Residual II }) \\
= & \sum_{t} w_{i t}\left(\hat{y}_{i t}-\bar{y}_{i}\right)^{2},
\end{aligned}
$$

where

$$
S S\left(\text { Residual II) }=\sum_{t} w_{i t}\left(y_{i t}-\hat{y}_{i t}\right)^{2},\right.
$$

the $\hat{y}_{i t}$ values in eqs. (14b) and (14c) being the predictions from Model II. These sums of squares are distributed approximately as chi-square variables with 1 and $(T-2)$ degrees of freedom, respectively.

Finally, if Model II does not provide an adequate fit, it is useful to examine Model III with different choices of elements for the vector $\mathbf{x}_{t}$. The estimation and testing strategy is again the same as in eqs. (9-11), but with the vectors $\boldsymbol{\theta}_{i}$ and $\mathbf{x}_{t}$ correspondingly reduced in dimension. Each age class can be modeled separately. Computer programs are available from the authors on request.

\section{GAINJ DEMOGRAPHIC DATA}

To illustrate the methods described above, we have applied them to census data collected among the Gainj of Papua New Guinea. The 
Gainj, who were studied by one of us (J.W.W.) in 1977 and 1978, number about 1500 individuals living in 20 villages in the vicinity of the Upper Tagui Valley on the northernmost fringes of Papua New Guinea's central highlands. They are classic swidden horticulturalists, cultivating sweet potato, taro, yams, and other crops in small, temporary plots cleared from primary or secondary rainforest. The density of the Gainj population is low by highland New Guinea standards, averaging only about 25 persons per square kilometer. Circumstantial evidence, summarized by Wood (1980), suggests that the Gainj have maintained essentially this same density for at least the last three to five generations. In 1969, however, the Gainj experienced an outbreak of A2 Hong Kong influenza, which was pandemic in Papua New Guinea at that time; as a result, they declined in number by about $6.5 \%$ between 1969 and 1970 . Since that time, the size of the Gainj population has returned to nearly its pre-epidemic level (Wood, 1980). It is this temporary disruption of the population that permits us to infer something about the demographic equilibrium. In effect, the epidemic serves as a natural perturbation experiment, and the aftermath of this disturbance provides us with the data analyzed in this paper.

These data (Table 1) were obtained from government census books for the Gainj Census Division; these books are kept at the Simbai Subdistrict Office in Madang Province and were kindly made available to us by Subdistrict Officers Moses Poi and George Chapok. A few words about the way government census patrols are conducted in Papua New Guinea will make clear both the strengths and limitations of these data. During the first such patrol, people from several neighboring villages are called together at some predetermined location, their names are recorded by household, and an estimated year of birth is assigned to each individual. On later visits, which occur at roughly yearly intervals, the list of names is read aloud. The names of persons who are no longer residing in the area are crossed out, a note being made as to whether they died or migrated out of the area. Similarly, new residents are recorded either as births or as in-migrants (or, in the years before complete enumeration has been achieved, as people who were missed by earlier censuses). In general, this strategy is an efficient one once the enumeration has become more or less complete, but it entails two potential sources of error. First, the ages assigned to individuals born before the first census are based solely on visual appearance and cannot be particularly accurate. Therefore we have employed only a very crude ten-year categorization of the adult population. Second, if a child is born after one census patrol and dies before the next, its existence may never be recorded. Consequently, the census procedure underestimates both the birth rate and the infant death rate to an extent determined by the length of the intercensal period. This has the effect of partially confounding fertility and infant survival in the analyses to follow, a point to which we shall return later.

The data in Table 1 pertain to only a part of the total Gainj population and were collected by government officers during five quasi-annual census patrols between 1970 and 1977 .

TABLE 1. Annual fertility $\left(\mathrm{f}_{\mathrm{it}}\right)$ and survival $\left(\mathrm{p}_{\mathrm{it}}\right)$ rates of Gainj females, listed by age class and census year, with age class sizes $\left(\mathrm{n}_{\mathrm{jt}}\right)$ in parentheses. Fertility rates refer strictly to the production of daughters

\begin{tabular}{|c|c|c|c|c|c|c|c|c|c|c|c|c|c|c|}
\hline \multirow{3}{*}{$\begin{array}{c}\text { Census } \\
\text { year } \\
(t)\end{array}$} & \multicolumn{14}{|c|}{ Age class in years (i) } \\
\hline & \multicolumn{2}{|c|}{$0-4$} & \multicolumn{2}{|c|}{ 5-9 } & \multicolumn{2}{|c|}{$10-19$} & \multicolumn{2}{|c|}{$20-29$} & \multicolumn{2}{|c|}{$30-39$} & \multicolumn{2}{|c|}{$40-49$} & \multicolumn{2}{|c|}{$50+$} \\
\hline & $f_{1 t}$ & $\left(n_{\mathrm{It}}\right)$ & $f_{2 t}$ & $\left(n_{2 t}\right)$ & $f_{3 t}$ & $\left(n_{3 t}\right)$ & $f_{4 t}$ & $\left(n_{4 t}\right)$ & $f_{5 t}$ & $\left(n_{5 t}\right)$ & $f_{6 r}$ & $\left(n_{6 t}\right)$ & $f_{7 t}$ & $\left(n_{7 t}\right)$ \\
\hline 1970 & - & (116) & - & $(82)$ & - & (184) & .115 & (157) & .112 & $(89)$ & .027 & $(74)$ & - & (58) \\
\hline 1972 & -- & (117) & - & $(97)$ & - & (187) & .131 & (160) & .118 & (102) & .014 & (73) & - & (69) \\
\hline 1974 & - & (116) & - & $(98)$ & - & (184) & .100 & (160) & .096 & (114) & .015 & (68) & - & (79) \\
\hline 1976 & - & (115) & - & $(100)$ & - & (174) & .111 & $(162)$ & .182 & (143) & .054 & (74) & - & (84) \\
\hline \multirow[t]{2}{*}{1977} & - & (121) & - & (109) & - & (183) & .093 & $(161)$ & .091 & $(143)$ & .014 & (71) & - & (85) \\
\hline & $p_{1 t}$ & $\left(n_{1 t}\right)$ & $p_{2 t}$ & $\left(n_{2 t}\right)$ & $p_{3 t}$ & $\left(n_{3 t}\right)$ & $p_{4 t}$ & $\left(n_{4 t}\right)$ & $p_{5 t}$ & $\left(n_{5 t}\right)$ & $p_{6 t}$ & $\left(n_{6 t}\right)$ & $p_{7 t}$ & $\left(n_{7 t}\right)$ \\
\hline 1970 & .983 & (116) & 1.000 & $(82)$ & .995 & (184) & .994 & $(157)$ & .989 & $(89)$ & 1.000 & $(74)$ & 983 & (58) \\
\hline 1972 & .889 & (117) & .959 & $(97)$ & 1.000 & (187) & .981 & $(160)$ & 1.000 & (102) & .932 & (73) & .986 & (69) \\
\hline 1974 & .974 & (116) & .990 & (98) & .989 & (184) & .981 & $(160)$ & .965 & (114) & .868 & (68) & .937 & (79) \\
\hline 1976 & .965 & (115) & .980 & $(100)$ & .994 & (174) & .957 & (162) & .944 & (143) & .905 & $(74)$ & .833 & (84) \\
\hline 1977 & .959 & $(121)$ & .991 & $(109)$ & .995 & (183) & .994 & (161) & .965 & (143) & .986 & (71) & .929 & (85) \\
\hline
\end{tabular}


Not all the censuses taken during this period have been included in this table, simply because the detailed breakdowns for some years were not available to us. A few earlier censuses were also available at Simbai, but comparison with our own pedigree data shows that these earlier efforts seriously underenumerated the extant population. Because the epidemic losses in 1969 presumably represent a different phenomenon from mortality induced by densitydependent factors, we exclude the 1969 census from the analysis as well. While the epidemic caused the pertubation, it is the aftermath that we examine for density-dependent effects.

The data for the five censuses included in Table 1 have been grouped into seven age categories, which we have labelled "early childhood" (roughly 0-4 years of age), "late childhood" ( $5-9$ years), "adolescent" (10-19 years), "early reproductive" (20-29 years), "mid-reproductive" (30-39 years), "late reproductive" (40-49 years) and "post-reproductive" (50+ years). We present the survival and fertility data for females only. Males have been excluded for two reasons: First, males often leave the area for irregular periods to work on lowland plantations, and the census data are such that it is difficult to determine accurately whether a given male was actually in residence within the Tagui Valley during any given intercensal period. Males who are not present contribute nothing to density stress and are not vulnerable to it. The females are permanent residents, however, and we are much more confident about both their numbers and survival rates. Second, the patrol officers conducting the censuses made little effort to ascertain paternity reliably, and the estimation of male fertility rates would be very difficult in practice. Female fertility is more easily ascertained, except for the under-reporting alluded to above, and an age-specific fertility table is easily constructed. We have elected to indicate only those births resulting in daughters, in accordance with the strictly single-sex models presented above.

The vital events listed in Table 1 occurred during the intercensal period following the year's census. As mentioned above, the duration of the intercensal period varies slightly from one year to the next. In Table 1, we have adjusted the fertility and survival rates to an annual basis by dividing the raw figures by the length of the intercensal period, on the assumption that births and deaths are uniformly distributed with respect to time over these short intervals. The adjustments are all small.
TABLE 2. Residual chi-squares (by census year and age class) for the fertility and survival rates of Gainj females after fitting Model I (density-independence)

\begin{tabular}{|c|c|c|c|c|c|c|}
\hline \multirow{2}{*}{$\begin{array}{l}\text { Age } \\
\text { class }\end{array}$} & \multicolumn{5}{|c|}{ Residual fertility chi-square } & \multirow{2}{*}{$\begin{array}{l}\text { Age class } \\
\text { total }\end{array}$} \\
\hline & 1970 & 1972 & 1974 & 1976 & 1977 & \\
\hline $0-29$ & & 0.670 & 0.180 & 0.000 & 0.487 & 1.362 \\
\hline-39 & 18 & 0.051 & 0.8 & 3.8 & 1.5 & 6.430 \\
\hline $0-49$ & 005 & 0.454 & & 1.377 & & 2.590 \\
\hline \multirow{2}{*}{$\begin{array}{l}\text { Age } \\
\text { class }\end{array}$} & \multicolumn{5}{|c|}{ Residual survival chi-square } & \multirow{2}{*}{$\begin{array}{l}\text { Age class } \\
\text { total }\end{array}$} \\
\hline & 1970 & 1972 & 1974 & 1976 & 1977 & \\
\hline $0-4$ & 771 & 6.0 & & 0.8 & & $11.940^{*}(4)$ \\
\hline $5-9$ & 076 & 1.55 & 0.4 & 0.004 & 0.6 & $\begin{array}{ll}78 & (4)\end{array}$ \\
\hline $10-19$ & 0.018 & 0.753 & 0.410 & 0.004 & 0.0 & 1.201 \\
\hline $20-29$ & 1.540 & 0.108 & & 2.5 & & (4) \\
\hline-39 & 1.266 & 2.34 & 0.0 & 1.169 & 0.030 & 4.823 \\
\hline $40-49$ & 3.602 & 0.227 & 1.860 & 0.088 & 3.479 & $9.256 \quad(4)$ \\
\hline $50+$ & 2.904 & 3.685 & 0.698 & 5.166 & 0.429 & $12.882^{*}$ \\
\hline
\end{tabular}

*Significant $(P<0.05)$.

+Number in parentheses $=$ degrees of freedom.

\section{RESULTS}

The first step of the analysis is to examine Model I, eq. (3), the density-independent null hypothesis. If the data fail to reject this most parsimonious hypothesis, we need proceed no further. The fertilities of the 20-29, 30-39, and 40-49 age classes were tested against Model $I$, and the results are presented in the top section of Table 2 . There is no evidence for a departure from the null hypothesis. In other words, there is no need to invoke density-dependent regulation of fertility to explain the results, and the analysis of fertility variation can be terminated. The resulting null hypothesis estimates of the fertilities of all seven age classes are: $0.000,0.000,0.000,0.113,0.129,0.035$, 0.000 .

Similarly, the survival figures for all seven age classes were tested against Model I, and these results are presented in the bottom section of Table 2. Here the outcome is mixed. Both the youngest $(0-4)$ and oldest $(50+)$ age classes deviate significantly from the null hypothesis of density-independent constancy, but none of the other age classes shows any significant departure from the null hypothesis. The "late reproductive" age class (40-49) does show a nonsignificant but suggestive departure from the null hypothesis $(0.10>P>0.05)$, but further analysis (not presented) shows no convincing evidence of any density-dependent pattern to the departures from constancy; we thus accept the density-independent model for this age class as well. For the $5-9,10-19, \ldots$, 40-49 age classes, the resulting null hypoth- 
esis estimates of the survival probabilities are: $0.974,0.991,0.974,0.959$, and 0.910 .

The next step is to examine Model II, eq. (4), for the youngest $(0-4)$ and oldest $(50+)$ age classes. The estimated $\alpha$ - and $\gamma$-coefficients and the chi-square test criteria are presented in Table 3, along with the corresponding figures for the Model I analysis. SS(Model II) $=0.42$ for the survival of the ( $0-4)$ age class; in other words, the youngest age class is not credibly damped by total population size. For the $(50+)$ age class, on the other hand, SS(Model II) = 4.98*, a significant improvement over the null hypothesis; there is thus some evidence for generalized damping on the survival of this age class. The residual variation for the $(50+)$ age class is nevertheless sigificant [SS(Residual II) $=7.90^{*}$, and so Model II is not entirely adequate here either.

To determine which age classes are responsible for the damping, it is necessary to test various versions of Model III, eq. (5). There are seven $\beta$-coefficents to work with, so there are $\left(\begin{array}{l}7 \\ 1\end{array}\right)+\left(\begin{array}{l}7 \\ 2\end{array}\right)+\ldots+\left(\begin{array}{l}7 \\ 7\end{array}\right)=127$ permutations of the model to be considered. Since there are only five census sets and one degree of freedom is required to fit each model, we can fit only models with fewer than five $\beta$-coefficients. That still represents $\left(\begin{array}{l}7 \\ 1\end{array}\right)+\left(\begin{array}{l}7 \\ 2\end{array}\right)+\ldots+\left(\begin{array}{l}7 \\ 4\end{array}\right)=98$ permutations to be tested. Although one would normally employ a stepwise version of the regression analysis in a situation such as this, a stepwise procedure is not guaranteed to converge on the best model. To avoid this problem, we have generated all 98 models and have searched for "sensible" sequences of models. In Table 3 we present the same set of models for both the $(0-4)$ and $(50+)$ age classes, because this set appears to represent the sensible solution in both cases.

In the case of the (0-4) age class, the (10-19) age class provides the most convincing evidence of damping, with $S S$ (Model III, $\beta_{13} \mid \alpha_{1}$ ) $=4.02 *$. The (20-29) age class, in contrast, yields no credible evidence of damping since SS(Model III, $\left.\beta_{14} \mid \alpha_{1}\right)=0.14$. Under either model, the residual variation remains significant [SS(Residual III $\left.\mid \alpha_{1}, \beta_{13}\right)=7.92^{*}$ and $S S$ (Residual III $\left.\mid \alpha_{1}, \beta_{14}\right)=11.80^{*}$ ], and we are justified in proceeding. The model with both the (10-19) and (20-29) age classes as dampers yields $S S$ (Model III, $\left.\beta_{13}, \beta_{14} \mid \alpha_{1}\right)=7.58^{*}$ and reduces the residual to $S S$ (Residual III $\mid \alpha_{1}, \beta_{13}$, $\left.\beta_{14}\right)=4.36$. For the $(50+)$ age class, either the $(10-19)$ or the (20-29) age class provides a good description of the observations, since $S S$ (Model III, $\left.\beta_{73} \mid \alpha_{7}\right)=10.86^{*}$ and SS(Model III, $\beta_{74} \mid$ $\left.\alpha_{7}\right)=9.85^{*}$. The coefficients for the model with the (10-19) age class as dampers are both negative, however, implying that survival of the $(50+)$ age class is very poor at low population density but improves with an increase in the numbers of women in the (10-19) age class. Since the dynamics of such a model would be pathological, we arbitrarily rule it out of consideration. Both coefficients for the model with the (20-29) age class as dampers are positive, and the residual variation [SS(Residual III ] $\left.\alpha_{7}, \beta_{74}\right)=3.03$ ] is nonsignificant. Although we are justified in stopping at this stage, we have also examined the model with both age classes as dampers. The coefficient for the (10-19) age class remains negative, however, and the improvement in $\boldsymbol{S S}$ (Model III) is small. On bal-

TABLE 3. Estimated parameters and sums of squares for Model I (density-independence), Model II (generalized density damping) and three versions of Model III (age-specific density damping) for the survival rates of females in age classes $(0-4)$ and $(50+)$. The three versions of Model III are indicated by the respective $\beta$-coefficients employed.

\begin{tabular}{|c|c|c|c|c|c|c|c|c|c|c|}
\hline \multirow[b]{3}{*}{ Model } & & & \multicolumn{4}{|c|}{ Estimated model parameters } & \multicolumn{4}{|c|}{ Source of survival variation } \\
\hline & \multirow{2}{*}{\multicolumn{2}{|c|}{$\begin{array}{l}\text { Damped } \\
\text { age class }\end{array}$}} & & & & & Mc & & Res & \\
\hline & & & $\hat{\alpha}_{i}$ & $\hat{\gamma}_{i}$ & $\beta_{i 3}$ & $\beta_{i 4}$ & SS & DF & SS & DF \\
\hline \multirow[t]{2}{*}{ I } & $0-4$ & $(i=1)$ & 2.760 & - & - & - & $\dagger$ & 1 & $11.94^{*}$ & 4 \\
\hline & $50+$ & $(i=7)$ & 2.237 & - & - & - & $\dagger$ & 1 & $12.88^{*}$ & 4 \\
\hline \multirow[t]{2}{*}{ II } & $0-4$ & $(i=1)$ & -0.304 & -0.0037 & - & - & 0.42 & 1 & $11.52^{*}$ & 3 \\
\hline & $50+$ & $(i=7)$ & 15.064 & 0.0152 & - & - & 4.98* & 1 & $7.90^{*}$ & 3 \\
\hline \multirow[t]{6}{*}{ III } & $0-4$ & $(i=1)$ & 18.609 & - & 0.0864 & - & $4.02^{*}$ & 1 & $7.92^{*}$ & 3 \\
\hline & $50+$ & $(i=7)$ & -20.901 & - & -0.1290 & - & $10.86^{*}$ & 1 & 2.02 & 3 \\
\hline & $0-4$ & $(i=1)$ & -5.176 & - & - & 0.0018 & 0.14 & 1 & $11.80^{*}$ & 3 \\
\hline & $50+$ & $(i=7)$ & 7.734 & - & - & 0.0796 & 9.85* & 1 & 3.03 & 3 \\
\hline & $0-4$ & $(i=1)$ & 85.921 & - & 0.1445 & 0.3535 & $7.58^{*}$ & 2 & 4.36 & 2 \\
\hline & $50+$ & $(i=7)$ & 24.079 & - & -0.0845 & 0.2298 & $11.78^{*}$ & 2 & 1.10 & 2 \\
\hline
\end{tabular}


ance, we accept the model with the (20-29) age class responsible for damping on the $(50+)$ age class. For both the damped age classes, (0-4) and $(50+)$, then, the best models take the form

$$
\begin{aligned}
& \hat{y}_{1 t}=85.921-0.144 n_{3 t}-0.354 n_{4 t} \\
& \hat{y}_{7 t}=7.734-0.080 n_{4 t}
\end{aligned}
$$

The corresponding predicted survival probabilities for the five census years are: $(0.979$, $0.912,0.941,0.971,0.929)$ for the $(0-4)$ age class and $(0.985,0.938 .0 .938,0.851,0.903)$ for the $(50+)$ age class. These predictions should be compared with the observed values of $(0.983$, $0.889,0.974,0.965,0.959)$ and $(0.983,0.986$, $0.937,0.833,0.929$ ), respectively.

Leaving aside the particular values of the estimates, the conclusions to be drawn from this analysis are fairly simple: (i) The survival of both the youngest (0-4) and oldest $(50+)$ age classes deviates significantly from the density-independent null hypothesis, and apparently both the "adolescent" (10-19) and "early reproductive" (20-29) age classes are implicated in the damping. (ii) Among the 5-9, 10-19, $20-29$, and $30-39$ age classes, survival shows no evidence of departure from density-independent constancy. (iii) The survival of the (40-49) age class shows a suggestive but nonsignificant departure from the null hypothesis, but lacking any convincing evidence for density-dependence, we accept the null hypothesis here as well. (iv) There is no evidence that fertility is anything but density-independent and constant. Recall that infant survival is confounded with reproduction in our data, a fact that could only have increased the apparent evidence for density-dependent fertility if infant survival itself were density-dependent. Thus the failure to detect any such pattern for fertility is doubly convincing.

It is important to recall a pair of statistical caveats when interpreting these results. First, a phenomenon such as density-dependence may be real without necessarily being statistically significant. Second, Type I error can never be avoided entirely, and so the null hypothesis will be rejected some percentage of the time even if it is true. Since we have only five census periods to work with, all of our conclusions must be tentative pending the availability of more data. In light of these facts, we have carefully avoided a statistical "fishing expedition," pursuing the alternative (density-dependent) hypothesis only when the null (density-independent) hypothesis clearly fails to account for the results. In each case, moreover, we have chosen the simplest density-dependent model consistent with the observations.

\section{DISCUSSION}

Among the Gainj, fertility and the survival of most age classes appear to be relatively insensitive to density effects. Only survival of the very young and the very old responds strongly in density-dependent fashion. We therefore predict that one major demographic effect of increasing population size is an increase in differential mortality among the young and elderly in comparison to individuals of intermediate ages. Population stress is experienced preferentially by the young and the old. If survival of the young and old is densitydamped, then individuals aged roughly 10-30 years appear to be the "dampers." We do not yet fully understand this finding, but we consider it important that: (i) the dampers are individuals experiencing sexual maturation and early reproduction, and (ii) reproductive individuals are themselves relatively insensitive to damping. It may well be that as the per capita availability of resources declines with increasing population size, food is consumed preferentially by adolescents and early reproductives at the expense of young children and post-reproductive adults.

In a later paper, we shall examine the question of whether this pattern of survival damping is sufficient to bring the population to a stable demographic equilibrium and, if so, what the predicted age structure at equilibrium will be. For the moment, however, we want to speculate about possible mechanisms of survival damping suggested by the observed age pattern. We know from a variety of biomedical studies that the Gainj population is a poorly nourished one, at least when it is near demographic equilibrium (Wood et al., 1979; Wood, 1980). We also know that the very young and old are the most poorly nourished segment of the population and suffer high overall mortality. Some $40 \%$ of infant deaths involve nutritional crises brought on by weanling diarrhea, and clinical and anthropometric signs of undernutrition are almost universal among children less than five years old. Additionally, there is a widespread hypochromic, microcytic anemia, apparently nutritional in etiology, that increases rapidly in prevalence with age, so that about $80 \%$ of all individuals over age 60 are affected. Moreover, the pattern of body fat development indicates that the "dampers" are individuals with proportionately very large fat 
reserves relative to younger and older individuals, reserves that are largely depleted over the course of the reproductive span (Johnson, 1981). Due to their marginal nutritional status, the young and old appear to be especially vulnerable to almost any kind of stress. A comparatively minor change in the environment, especially in the availability of food, would be sufficient to compromise their chances of survival. There is little doubt, then, that the density-dependent survival rates we have demonstrated among the Gainj are a reflection of resource (food) limitation.

In this paper, we have developed a method for detecting density-dependent variation in fertility or mortality, using the kind of limited longitudinal data that is likely to be available to many anthropologists. For the Gainj, the results suggest that fertility is density-independent but that mortality is partially densitydependent, suggesting in turn that population size is regulated by mortality rather than fertility in this particular case. The generality of this conclusion can be assessed only by application of the method to other populations. We do not wish to prejudice future results at this stage by arguing that either fertility or mortality is inherently more likely to be implicated in population regulation. We do, however, wish to point out that the issue is still very much an open one.

\section{ACKNOWLEDGMENTS}

We thank the staff of the Simbai Subdistrict Office, Madang Province, Papua New Guinea, especially Moses Poi and George Chapok, for providing access to the Gainj census material. We also thank Ranajit Chakraborty, Alexander Gimmelfarb, Peter Grant, Patricia Johnson, Frank Livingstone, Jeffrey Long, James Neel, Edward Pollak, Virginia Vitzthum, and Kenneth Weiss for discussion of methods and results, and Wanda Liu for her superb skills in helping to program the estimation procedures. J.W.W.'s field research in New Guinea was partially supported by NSFBNS-77-01499. This study was further supported by grants NIH-5-T32-GM-08123-06 (to J.W.W.) and NSF-DEB-7823293 (to P.E.S.).

\section{LITERATURE CITED}

Benedict, B (1972) Social regulation of fertility. In GA Har ison and AJ Boyce (eds): The Structure of Human Populations. London: Oxford University Press, pp. 73-89.

Charlesworth, B (1980) Evolution in Age-Structured Populations. London: Cambridge University Press.

Dumond, DE (1975) The limitation of human population: a natural history. Science 187:713-721.

Gart, JJ, and Zweifel, JR (1967) On the bias of various estimates of the logit and its variance with application to quantal bioassay. Biometrika 54:181-187.

Halberstein, RA (1980) Population regulation in an island community. Hum. Biol. 52:479-498.

Hayden, B (1972) Population regulation among hunter/ gatherers. World Archaeol. 4:205-221.

Johnson, PL (1981) When dying is better than living: female suicide among the Gainj of Papua New Guinea. Ethnology $20: 325-334$

Leslie, PH (1959) The properties of a certain lag type of population growth and the influence of an external random factor on a number of such populations. Physiol. Zool. 32:151-159.

Neter, J, and Wasserman, W (1974) Applied Linear Statistical Models. Homewood, Ill: RD Irwin

Pielou, EC (1977) Mathematical Ecology. New York: Wiley.

Rao, CR (1973) Linear Statistical Inference and Its Applications. New York: Wiley.

Smouse, PE, and Weiss, KM (1975) Diserete demographic models with density-dependent vital rates. Oecologia $21: 205-218$

Weiss, KM, and Smouse, PE (1976) The demographic stability of small human populations. J Hum. Evol. 5:59-73.

Wood, JW (1980) Mechanisms of Demographic Equilibrium in a Small Human Population, the Gainj of Papua New Guinea. Doctoral dissertation, University of Michigan, Ann Arbor, Michigan.

Wood, JW, Johnson, PL, Coveney, J, and Nurse, GT (1979) Nutrition, growth and childhood mortality in the Tagui Valley, Madang Province, Papua New Guinea. Unpublished manuscript. 